\title{
Gamma-rays from millisecond pulsar binary systems
}

\author{
Wlodek Bednarek* \\ Department of Astrophysics, University of Łódź \\ E-mail: bednar@uni.lodz.pl
}

\begin{abstract}
We consider the interaction of a millisecond pulsar (MSP) wind with a very inhomogeneous stellar wind from the companion star within binary systems of the black widow and redback types. Our aim is to determine the features of $\gamma$-ray emission produced in the collision region of the winds from a few typical MSP binary systems. Electrons accelerated in such mixed, turbulent winds can interact with strong radiation from the companion star, producing $\gamma$-rays in the inverse Compton process, fluxes of which are expected to be modulated on the periods of the binary systems. Applying numerical methods, we calculated the GeV-TeV gamma-ray angle dependent spectra expected from some millisecond pulsar binary systems. It is concluded that energetic millisecond pulsar binary systems might create a new class of $\mathrm{TeV} \gamma$-ray sources that could be detectable by the future Cherenkov arrays (e.g., CTA) and possibly also by the extensive campaigns with the present arrays.
\end{abstract}

Frontier Research in astrophysics,

26 - 31 May, 2014

Mondello (Palermo), Italy

${ }^{*}$ Speaker. 


\section{Introduction}

Most of the millisecond pulsars are members of binary systems. The masses of companion stars are a few percent of the solar mass in the case of black widow binaries and a few tens percent of solar mass in the case of redback binaries (Roberts 2012). The energy realized by the millisecond pulsar heats the surface of the companion star to temperature significantly above the temperature of the side of the star opposite to the pulsar (Breton et al. 2013). It is expected that electrons can be accelerated within the MSP binaries at the shock that appears in the interaction of winds from the millisecond pulsar and the companion star.

In this paper we consider a specific model for the interaction of winds from the MSP and the companion star. In contrast to previous models (Harding \& Gaisser 1990, Arons \& Tavani 1993), we assume that the wind from the companion star is highly inhomogeneous. Then, the pulsar and stellar winds should mix very efficiently at the location where the pressure of both winds becomes comparable. The mixed winds expand from the binary system with a relatively low velocity owing to the barion loading of the relativistic pulsar wind with the matter from the relatively slow stellar wind. Relativistic electrons, which are captured in such mixed winds, can interact efficiently with the radiation from the nearby companion star producing $\mathrm{TeV} \gamma$-rays. We calculate the $\gamma$-ray and synchrotron spectra, produced by these electrons and compare them with the available X-ray observations from these binary systems. Based on this comparison, the $\mathrm{TeV} \gamma$-ray fluxes are predicted (for details see Bednarek 2014).

\section{Interaction of winds in millisecond pulsar binary systems}

We modify the standard scenario for processes in MSP binary systems (Harding \& Gaisser 1990, Arons \& Tavani 1993) by assuming that the wind from the companion star is highly inhomogeneous. In this case, the interaction of winds produces a very turbulent mixed pulsar-stellar wind that moves together with the velocity,

$$
v_{\text {mix }}=\left[\frac{2 L_{\mathrm{pul}} \Delta \Omega_{\mathrm{pul}}}{\dot{M}_{\star} \Delta \Omega_{\star}}\right]^{1 / 2} \approx 4 \times 10^{9}\left(\frac{\xi_{-1} L_{35}}{M_{-11}}\right)^{1 / 2} \frac{\mathrm{cm}}{\mathrm{s}}
$$

where $L_{\text {pul }}=10^{35} L_{35} \mathrm{erg} \mathrm{s}^{-1}$ is the power of the pulsar wind, $\dot{M}=10^{-11} M_{-11} \mathrm{M}_{\odot} \mathrm{yr}^{-1}$ is the mass loss rate of the companion star, and $\Delta \Omega_{\text {pul }}$ and $\Delta \Omega_{\star}$, describe a part of the solid angles that are over-taken by the collision region as observed from the pulsar site and from the companion star site, respectively. The above estimated relatively low velocity of the mixed winds guarantees that relativistic electrons stay relatively close to the stellar surface for long enough time that their interactions with soft radiation from the stellar surface is efficient.

We consider the role of the high-energy processes that are expected to be important in the general scenario discussed above. We are interested in processes which could explain X-ray emission produced within the MSP binary systems of the PSR B1957+20 type. Such synchrotron X-ray emission can be produced within the binary system when the relativistic electrons move in the region of turbulent, mixed MSP-stellar wind. In fact, the phase dependent X-ray emission is observed up to at least $\varepsilon_{\text {syn }} \sim 8 \mathrm{keV}$ from this binary system (Huang et al. 2012). The magnetic field in the emission region and the Lorentz factor of electrons should fulfil the following condition,

$$
\varepsilon_{\mathrm{syn}} \approx m_{\mathrm{e}} c^{2}\left(B / B_{\mathrm{cr}}\right) \gamma_{\mathrm{e}}^{2}
$$


where $m_{\mathrm{e}} c^{2}$ is the electron rest energy, $B$ the magnetic field strength in the emission region, $B_{\mathrm{cr}}=$ $4.4 \times 10^{13} \mathrm{G}$ is the critical magnetic field strength, and $\gamma_{\mathrm{e}}$ the Lorentz factor of electrons. The magnetic field strength in this region can be estimated by a simple extrapolation from the pulsar surface, assuming the dipole structure of the magnetic field below the light cylinder and the toroidal structure in the pulsar wind region,

$$
B_{\text {sh }}=3 \sigma^{1 / 2} B_{\text {pul }}\left(\frac{R_{\text {pul }}}{R_{\mathrm{LC}}}\right)^{3} \frac{R_{\mathrm{LC}}}{\rho_{\mathrm{o}}} \approx \frac{0.3 \sigma_{-4}^{1 / 2} B_{8}}{P_{2}^{2} \rho_{11}} \mathrm{G},
$$

where $B_{\mathrm{pul}}=10^{8} B_{8} \mathrm{G}$ and $P_{\mathrm{pul}}=2 P_{2} \mathrm{~ms}$ are the surface magnetic field strength and the period of the pulsar, $R_{\mathrm{LC}}=c P_{\mathrm{pul}} / 2 \pi$ is the light cylinder radius of the pulsar, $\sigma=10^{-4} \sigma_{-4}$ is the magnetization parameter of the pulsar wind, $\rho_{o}=a-R_{\mathrm{Sh}} R_{\star}=10^{11} \rho_{\mathrm{o}} \mathrm{cm}$ is the distance of the wind collision region from the MSP, $R_{\mathrm{Sh}}$ is the distance of the shock from the companion star, $R_{\star}$ is the radius of the star, and $a$ is the semimajor axis of the binary system. The magnetic field is expected to reconnect very efficiently in the highly turbulent region of the mixed winds. Therefore, the magnetic field strengths, described by very low values of the magnetization parameter, $\sigma \ll 10^{-2}$, should not be surprising. For the above value of the magnetic field at the transition region (Eq. 2.3), the Lorentz factor of electrons, have to be at least,

$$
\gamma_{\mathrm{e}} \sim 1.5 \times 10^{6} P_{2} \rho_{11}^{1 / 2} /\left(\sigma_{-4}^{1 / 4} B_{8}^{1 / 2}\right),
$$

to produce the observable X-ray emission in synchrotron process in the case of PSR B1957+20 (Eq. 2).

The maximum energies of electrons accelerated at the transition region are determined by the acceleration time scale and the time scales for the energy losses or the escape from the acceleration region. The acceleration time scale can be estimated from $\tau_{\text {acc }}=R_{\mathrm{L}} /(c \chi) \approx 1 E /\left(\chi_{-1} B\right) \mathrm{s}$, where the electron energy is in $\mathrm{TeV}$ and $\chi=0.1 \chi_{-1}$ is the acceleration parameter. We assume that the acceleration parameter can be related to the velocity of the mixed winds in the following way: $\chi \sim\left(v_{\text {mix }} / c\right)^{2}=0.1 v_{10}^{2}$, where the mixed wind velocity is $v_{\text {mix }}=10^{10} v_{10} \mathrm{~cm} \mathrm{~s}^{-1}$. The maximum energies of electrons might be constrained by their escape from the acceleration region with the velocity of the mixed pulsar/stellar wind. The advection time scale of the mixed wind is $\tau_{\mathrm{adv}}=$ $\pi R_{\mathrm{sh}} R_{\star} / v_{\text {mix }} \approx 10 R_{11} / v_{10} \mathrm{~s}$, where $R \approx \pi R_{\mathrm{sh}} R_{\star}=10^{11} R_{11} \mathrm{~cm}$ is the characteristic distance scale for the propagation of the turbulent wind around the companion star. By comparing this time scale with the acceleration time scale, we get the limit on the electron energies,

$$
E_{\mathrm{adv}}^{\max } \approx 3 R_{11} \sigma_{-4}^{1 / 2} B_{8} v_{10} /\left(P_{2}^{2} \rho_{11}\right) \mathrm{TeV} .
$$

To fully understand the importance of the energy losses of accelerated electrons, we compare the electron acceleration time scale with the synchrotron or the inverse Compton energy-loss time scales. The synchrotron energy-loss time scale can be calculated from $\tau_{\mathrm{syn}}=E_{\mathrm{e}} / \dot{E}_{\mathrm{syn}} \approx 370 /\left(B^{2} E\right)$ s. For the parameters of considered binary systems, the IC energy losses in the Thomson regime usually dominate the synchrotron energy losses. However, the IC energy losses in the KleinNishina (KN) regime declines, and the synchrotron losses can start to dominate at high energies. In this case, the maximum energy of accelerated electrons is determined by the balance between the acceleration time scale and the synchrotron time scale. They are estimated on

$$
E_{\mathrm{syn}}^{\max } \approx 19\left(\chi_{-1} / B\right)^{1 / 2} \mathrm{TeV} .
$$


Then, electrons can reach energies as large as (see Eq. 3),

$$
E_{\mathrm{syn}}^{\max } \approx 35 P_{2} v_{10} \rho_{11}^{1 / 2} /\left(\sigma_{-4}^{1 / 4} B_{8}^{1 / 2}\right) \mathrm{TeV} .
$$

We now estimate the electron energy at which synchrotron losses become comparable to the IC losses in the KN regime. For the IC energy loss time scale in the KN regime, we apply the approximate formula by assuming that the IC energy losses in the KN regime are comparable to the IC energy losses on the border between $\mathrm{T}$ and $\mathrm{KN}$ regimes (see e.g. Eq. 7 in Bednarek 2011). Then, the IC losses in $\mathrm{KN}$ regime can be estimated from

$$
\tau_{\mathrm{IC}}^{\mathrm{KN}}=\frac{3 m_{\mathrm{e}}^{2} c^{4} E}{4 c \sigma_{\mathrm{T}} U_{\mathrm{rad}} E_{\mathrm{T} / \mathrm{KN}}^{2}} \approx \frac{16.7 E R_{\mathrm{sh}}^{2}}{T_{4}^{2}} \mathrm{~s},
$$

where $\sigma_{\mathrm{T}}$ is the Thomson cross section, $U_{\text {rad }}$ is the density of stellar radiation at the acceleration region, $T=10^{4} T_{4} \mathrm{~K}$ is the surface temperature of the companion star, and $E_{\mathrm{T} / \mathrm{KN}}=\left(m_{\mathrm{e}} c^{2}\right)^{2} / 3 k_{\mathrm{B}} T \approx$ $0.1 / T_{4} \mathrm{TeV}$ is the electron energy corresponding to transition between the $\mathrm{T}$ and $\mathrm{KN}$ regimes. By comparing $\tau_{\mathrm{IC}}^{\mathrm{KN}}$ with $\tau_{\mathrm{syn}}$, we can estimate the critical energy below which electrons lose energy mainly in the IC process,

$$
E_{\mathrm{syn}}^{\mathrm{IC} / \mathrm{KN}} \approx 4.7 \frac{T_{4}}{R_{\mathrm{sh}} B_{\mathrm{sh}}} \approx 16 \frac{T_{4} P_{2}^{2} \rho_{11}}{\sigma_{-4}^{1 / 2} B_{8} R_{\mathrm{sh}}} \quad \mathrm{TeV} .
$$

This critical electron energy is usually not far from the maximum electron energy determined from the comparison of the acceleration time scale and the synchrotron energy-loss time scale.

We conclude that the most important process, which determines the maximum energies of electrons accelerated in the turbulent region, is the advection from the acceleration region. However, electrons can lose a significant amount of their energy on radiation processes, such as the IC scattering of soft photons from the companion star and the synchrotron radiation in the magnetic field in the colliding wind region.

\section{Gamma rays from comptonization of stellar radiation}

A few MSP binary systems (shown in Table 1 in Bednarek 2014) have been selected for detailed calculations due to their well known parameters of the pulsars and binary systems. The injection place of relativistic electrons is located outside the isotropic source of stellar radiation, therefore the IC scattering process has to occur anisotropically because the electron-photon collision rate depends on the angle between the direction of propagation of the electron and the soft photon. To calculate the $\gamma$-ray spectra expected in terms of our model, we applied the Monte Carlo code that follows the IC $e^{ \pm}$pair cascade processes (see e.g. Bednarek 2006). We calculated the $\gamma$ ray spectra from the IC process and the synchrotron spectra produced by these same electrons. We assume that electrons are injected with the differential power law spectrum (spectral index -2), extending to the maximum energies allowed by their escape from the binary system. The synchrotron spectra were also calculated. In contrast to the IC spectra, the synchrotron spectra are isotropic, since the magnetic field and electron distribution in the mixed wind reference frame are assumed to be isotropic. 
Figure 1: Comparison of the high-energy emission (energy flux versus energy) expected from the MSP binary systems with the sensitivities of Fermi-LAT (10 yrs - extragalactic background, see thin dashed curve, Funk et al. 2013), MAGIC (thin dotted curve, Aleksić et al. 2012) and CTA (thin dot-dashed, Bernlöhr et al. 2012), for different ranges of observation angles: $0.9 \leq \cos \beta \leq 1.0$ (dot-dashed, away from the companion star), $0.5 \leq \cos \beta \leq 0.6$ (dotted), $-0.1 \leq \cos \beta \leq 0$. (dashed), $-0.5 \leq \cos \beta \leq-0.4$ (solid), $-1.0 \leq \cos \beta \leq-0.9$ (dot-dot-dashed). The other parameters of the models have been fixed on $\sigma=10^{-4}$, and $v_{\mathrm{adv}}=10^{10} \mathrm{~cm} \mathrm{~s}^{-1}$, and $E_{\mathrm{e}}$ is given by Eq. 7 . The spectra are normalized to the X-ray fluxes observed from PSR B1957+20 (Huang et al. 2012), PSR J1023+0038 (Bogdanov et al. 2011), PSR J1810+1744 (Gentile et al. 2013). For PSR J1816+4510, the upper limit derived from the Swift data (Kaplan et al. 2012). The spectra have been calculated for the energy conversion efficiencies from pulsars to relativistic electrons estimated on $1.6 \times 10^{-2}$ (for PSR J1023+0038), $6.5 \times 10^{-2}$ (PSR J1810+1744), $5 \times 10^{-3}$ (PSR J1816+4510), and $1.8 \times 10^{-3}($ PSR B1957+20).

The X-ray emission, modulated with the period of the binary system, has been reported from three of these selected MSP binary systems: PSR B1957+20 (Huang et al. 2012), PSR J1023+0038 (Bogdanov et al. 2011), and PSR J1810+1744 (Gentile et al. (2013). For PSR J1816+4510, only the upper limit on the X-ray flux has been reported based on the Swift data (Kaplan et al. 2012). These $\mathrm{X}$-ray positive detections (and the upper limit) are used for the normalization for the synchrotron $\mathrm{X}$-ray emission calculated in terms of our model. Such procedure allows us to predict the absolute level of the $\mathrm{TeV} \gamma$-ray emission.

The synchrotron and IC $\gamma$-ray spectra, calculated for likely parameters defining considered model, were compared with the observations of the modulated synchrotron emission in the X-ray energy range. We apply the velocity of the mixed pulsar-stellar wind equal to $10^{10} \mathrm{~cm} \mathrm{~s}^{-1}$. For the assumed magnetization parameter, $\sigma=10^{-4}$, and the other typical parameters of the pulsar and its binary system, we estimated the maximum energies of accelerated electrons by using Eq. 7. In Fig. 1 we show the expected IC $\gamma$-ray spectra produced by electrons with the differential power-law spectrum (spectral index -2), after normalization to the observed modulated X-ray emission from binary systems, PSR J1023+0038, PSR J1810+1744, and PSR B1957+20, and consistent with the 
upper limit derived for the binary system J1816+4510. Specific curves show the results for different ranges of the cosine of the observation angles, i.e., related to different phases of the binary system. The IC $\gamma$-ray spectra in the sub-TeV energy range are close to the level of sensitivity of the present Cherenkov telescopes (such as HESS, MAGIC, and VERITAS), for the observation angles that are optimal for efficient $\gamma$-ray production (i.e., pulsar behind the companion star).

In the case of three binary systems, PSR B1957+20, PSR J1023+0038, and PSR J1810+1744, the estimates of the inclination angles are available in the literature: $65^{\circ}$ for PSR B1957+20 (Reynolds et al. 2007) and $46^{\circ}$ for PSR J1023+0038 (assuming that the neutron star mass is equal to the 1.4 solar masses, Archibald et al. 2009, Archibald et al. 2010), and $48^{\circ}$ for PSR J1810+1744. We conclude that the $\mathrm{TeV} \gamma$-ray signals from these binary systems have a chance to be detected in the extensive observations with the present Cherenkov telescopes only during specific range of orbital phases. The situation should be much better with the construction of the next generation of Cherenkov telescope arrays such as CTA. In the case of CTA, the TeV $\gamma$-ray emission from MSP binary systems is expected to be probed for most of the range of orbital phases. Modulated $\mathrm{GeV}$ $\gamma$-ray emission from the considered binary systems is not likely to be detected in the Fermi-LAT data. The exception can only be the redback-type binary, PSR J1816+4510, which contains an exceptionally luminous companion star with surface temperature above $\sim 10^{4} \mathrm{~K}$ (Kaplan et al. 2013).

\section{Conclusion}

We have considered the high energy processes that are expected to occur within the MSP binary systems of the redback and black widow types. We modified the general scenario proposed for those objects by assuming that the wind of the companion star is very inhomogeneous. Therefore, winds should mix efficiently at the collision region and move with velocity that is clearly lower than the velocity of light. Also, the reconnection of the magnetic field should be very efficient in such a case. As a consequence, a relatively low value of the magnetic field is expected at the transition region. Since the mixed winds move relatively slowly, relativistic electrons stay a relatively long time in the dense radiation of the companion star, losing energy on the IC scattering process. Thanks to the relatively weak magnetic field at the transition region, the cooling process of relativistic electrons is not completely dominated by synchrotron radiation.

As an example, we applied our model to the four MSP binary systems, two of the black widow type and two of the Redback type. We calculated the synchrotron and IC $\gamma$-ray spectra escaping to the observer located at different angles in respect to direction defined by the stars. From normalization of calculated synchrotron spectra to the observed non-thermal X-ray flux from these binary systems, we predicted the absolute level of the $\mathrm{TeV} \gamma$-ray flux from these binaries. It is concluded that the $\mathrm{TeV}$ emission is comparable to the sensitivities of the present Cherenkov telescopes. However, detailed investigation of this $\mathrm{TeV}$ emission over the whole range of phases will be only possible with the construction of the CTA.

\section{Acknowledgments}

This work is supported by the grant through the Polish Narodowe Centrum Nauki No. 2011/01/B/ST9/00411. 


\section{References}

[1] Aleksić, J. et al. (MAGIC Collaboration) 2012 APh 35, 435

[2] Archibald, A.M., Stairs, I.H., Ransom, S.M. et al. 2009 Science 324, 1411

[3] Archibald, A.M., Kaspi, V. M., Bogdanov, S. et al. 2010 ApJ 722, 88

[4] Arons, J., Tavani, M. 1993 ApJ 403249

[5] Bednarek, W. 2006 MNRAS 368, 579

[6] Bednarek, W. 2011 MNRAS 418, L49

[7] Bednarek, W. 2014 A\&A 561, A116

[8] Bernlöhr, K. et al. (CTA Consortium) 2013 APh 43, 171

[9] Bogdanov, S., Archibald, A.M.; Hessels, J.W.T. et al. 2011 ApJ 742, 97

[10] Breton, R.P., van Kerkwijk, M.H., Roberts, M.S.E. et al. 2013 ApJ 769, 108

[11] Funk, S., Hinton, J.A. (CTA Consortium) 2013 APh 43, 348

[12] Gentile, P., Roberts, M., McLaughlin, M. et al. 2013 ApJ, submitted (arXiv:1305.6799)

[13] Harding, A.K., Gaisser, T.K. 1990 ApJ 358, 561

[14] Huang, R.H.H. Kong, A.K.H., Takata, J., Hui, C.Y., Lin, L.C.C., Cheng, K.S. 2012 ApJ 760, 92

[15] Kaplan, D.L., Stovall, K., Ransom, S.M. et al. 2012 ApJ 753, 174

[16] Roberts, M.S.E. in Proc. Neutron Stars and Pulsars: Chellenges and Opportunities after 80 years", ed. J. van Leeuwen (2012) IAUS 291, arXiv:1210.6903

\section{DISCUSSION}

GENNADY BISNOVATYI-KOGAN: I do not understand how you come to the picture of colliding winds in the binary NS+WD or NS+RG! In the last case you have an X-ray source, accretion, and no pulsar wind. In the radio MS pulsars the WD does not produce wind. The induced evaporation by the pulsar wind does not produce collision of winds: if the pulsar wind would be stopped by the shock, the evaporation should stop.

WLODEK BEDNAREK: We do not consider in the paper NS binaries which contain slowly rotating, and accreating, neutron star and stellar companion but only specific type of NS binary systems which contain radio MSP pulsar and low mass WD companion (binaries of the black widow and redback type).

A prototype of such specific binary systems is black widow binary containing MSP PSR B1957+20. The presence of a shock in this binary system is expected since its discovery, since the pulsed radio emission shows an eclipse lasting for about $10 \%$ of orbital phase (Fruchter et al. 1988). The emission from such systems has been popularly modeled in the scenario of the colliding MSP and stellar winds within the binary (e.g. Arons \& Tavani 1993). The companion star is expected to be evaporated by the energy realised from the inner MSP magnetosphere (e.g. Ruderman et al. 1989). It is expected that due to this process, the companion stars in the MSP black 
widow type binaries have masses of only a few percent of the Solar mass. The average evaporation rate (companion star wind mass loss rate) during the characteristic age of 10- Gyrs is expected to be of the order of $10^{-10} \mathrm{M}_{\odot} \mathrm{yr}^{-1}$, as assumed in our work. 